Meta

Journal des tradlucteurs

Translators' Journal

\title{
Détenteur et titulaire
}

\section{Carlos Del Burgo}

Volume 24, numéro 3, septembre 1979

URI : https://id.erudit.org/iderudit/002507ar

DOI : https://doi.org/10.7202/002507ar

Aller au sommaire du numéro

Éditeur(s)

Les Presses de l'Université de Montréal

ISSN

0026-0452 (imprimé)

1492-1421 (numérique)

Découvrir la revue

Citer cet article

Del Burgo, C. (1979). Détenteur et titulaire. Meta, 24(3), 370-373.

https://doi.org/10.7202/002507ar

Ce document est protégé par la loi sur le droit d'auteur. L'utilisation des services d'Érudit (y compris la reproduction) est assujettie à sa politique d'utilisation que vous pouvez consulter en ligne.

https://apropos.erudit.org/fr/usagers/politique-dutilisation/
Cet article est diffusé et préservé par Érudit.

Érudit est un consortium interuniversitaire sans but lucratif composé de l’Université de Montréal, l'Université Laval et l'Université du Québec à Montréal. Il a pour mission la promotion et la valorisation de la recherche. https://www.erudit.org/fr/ 


\section{DÉTENTEUR ET TITULAIRE *}

\section{Aperçu de la question}

On se trouve face à deux termes qui deviennent difficiles à différencier tant leurs domaines d'application sont voisins. On utilise parfois à tort les deux vocables «détenteur» et «titulaire » comme de parfaits synonymes et l'on s'efforce dans d'autres cas de les distinguer l'un de l'autre là où il n'y a pas lieu de le faire. Voici quelques cas concrets qui permettront de mieux saisir la question.

\section{« Détenteur *}

Dans les domaines de l'enseignement ou des loisirs, on dit de certaines personnes qu'elles sont détentrices d'un prix ${ }^{14}$ (à un concours). Dans celui des affaires et du droit, on peut être détenteur ou porteur (v. observation ci-dessous) d'actions (actionnaire), d'obligations (obligataire), d'effets de commerce, de traites, de chèques, de titres, de lettres de change, de créances et de devises, entre autres. On retrouve dans ces mêmes secteurs d'autres syntagmes construits avec le terme « détenteur », tels détenteur d'une succession ${ }^{8}$, tiers détenteur ${ }^{8}$.

Dans d'autres domaines, quelqu'un peut être détenteur d'un billet gagnant ${ }^{5}$ (tirage au sort), d'un pouvoir ${ }^{8}$ (politique ou autre) ou d'un record ${ }^{9}$ (sports).

\section{Observation sur «porteur»}

Dans la langue courante, un porteur est soit « une personne qui porte quelque chose sur soi, qui est en possession de» ( on l'a trouvé porteur d'un

\footnotetext{
Les numéros mentionnés renvoient à la bibliographie.
} 
couteau à cran d'arrêt ${ }^{\circ} \gg$ ), soit une (personne qui est chargée de remettre une lettre, une dépêche, etc. ${ }^{\circ}$ ». Fait à signaler : "porteur * revêt dans certains contextes un sens plus spécialisé, signifiant alors « celui qui détient un titre n'indiquant pas le titulaire du droit ${ }^{13}$.

Ex. : titre au porteur (par opposition à a titre nominatif , et

4 titre à ordre ${ }^{13} \gg$ )

obligation au porteur

payable au porteur

payez au porteur

(On n'entrera pas ici dans le détail des autres acceptions de \$ porteur ».)

\section{s Titulaire,}

Il est à noter que, dans le langage administratif, la polysémie du terme « titulaire * provient du fait que \& titre » revêt lui-même plusieurs acceptions, prenant d'abord le sens d'aptitudes, qualités, services, diplômes, situations particulières d'un candidat ${ }^{5}$, puis celui d'\& écrit, document établissant un droit social $\gg\left(c .-a ̀-d\right.$. un certificat $\left.{ }^{9}\right)$. Il s'ensuit que le titulaire d'un poste est celui « qui a une fonction, une charge pour laquelle il a été personnellement nommé, en vertu d'un titre ${ }^{14} \gg$. On sait également que, dans la fonction publique, e un fonctionnaire est titulaire lorsque, possédant les titres nécessaires et ayant satisfait aux conditions d'âge et de stage réglementaires, il a été régulièrement nommé à la fonction qu'il exerce. S'oppose à : stagiaire, auxiliaire, intérimaire, remplaçant, surnuméraire, contractuel ${ }^{5}$. 》

Ailleurs, c'est la loi ou le succès à une épreuve quelconque qui font qu'une personne est titulaire d'un droit ou d'un permis de conduire, par exemple. "Les vieux travailleurs sont titulaires d'une pension (en possession du titre qui leur permet d'en bénéficier) ${ }^{5}$.

Dans le secteur des affaires et du droit, il apparaît cependant qu'écrire * titulaire d'une action \$ (ou d'une obligation) n'est pas très heureux, en dépit de ce qu'en disent certains lexicographes bilingues. C'est plus précisément parce qu'un actionnaire est détenteur (ou porteur) d'actions qu'il devient titulaire de certains droits, d'où une relation de cause à effet (être titulaire d'une action signiflerait, à proprement parler, \&y avoir droit», ce qui jette un éclairage différent sur la réalité dans laquelle on désire simplement faire état du possesseur de l'action, en dehors de toute considération juridique). Par ailleurs, l'usage tiendra compte, dans d'autres cas, de la différence marquée entre titulaire et détenteur; ainsi, le détenteur d'un billet n'en sera pas nécessairement le titulaire, puisqu'il devra encore remplir certaines conditions avant d'être le propriétaire effectif de ce billet.

\section{Résumé}

Il ressort de cette étude que le trait sémantique qui différencie «titulaire \$ de a détenteur \$ (et de « porteur ») réside en l'existence d'une condition particulière, condition à laquelle il faut satisfaire pour avoir droit à l'appellation de 
《 titulaire ». En effet, s'il suffit au détenteur (ou porteur) d'un document, d'un chèque, d'entrer en possession de ces éléments - à la suite d'un achat ou d'une cession, par exemple - le titulaire du permis de conduire sera, en fait, l'objet d'une sélection, puisqu'il devra démontrer une capacité à maîtriser son véhicule (le titulaire d'un emploi devra, lui, prouver que ses aptitudes, son attitude ou son expérience isont adéquates).

Carlos Del Burgo

\section{BIBLIOGRAPHIE}

1. ANDERLA, G., SCHMIDT-ANDERLA, G., Dictionnaire des affaires, Paris, Delmas,

2. BELL CANADA, Centre de terminologie, Fichier général, Montréal.

3. BELL CANADA, Service du commercial, Lexique général du commercial, édition
revue et augmentée, Montréal, 1978, 155p.

4. BÉNAC, H., Dictionnaire des synonymes, Paris, Hachette, 1965, 1026 p.

5. DAVAU, M., COHEN, M., LALLEMAND, M., Dictionnaire du français vivant, Paris,
Bordas, 1972, 1338 p. 6. JERAUTE, J., Vocabulaire français-anglais et anglais-francais de termes. et locutions
juridiques, Paris, Librairie gênérale de droit et de jurisprudence, 1952,415 p.

7. KETTRIDGE, J.O., Dictionnaire français-anglais, anglais-français de termes, locutions
et pratique de commerce et de finance, Londres, Routledge \& Kegan Paul, 1965, 647

8. LAROUSSE, Grand Larousse encyclopédique, Paris,

9. LAROUSSE, Lexis, Paris, 1975, 1950 p.

10. LEMEUNIER, F., Dictionnaire juridique. économique et financier, (documents actuels),
Paris, Delmas, 1969, 366 p.

11. LITTRE, É., Dictionnaire de la langue française, Paris, Gallimard, 1966, 7 vol.

12. QUEMNER, T.A., Dictionnaire juridique français-anglais, Paris, Navarre, 1969, 1 vol. 13. ROBERT, P., Dictionnaire alphabétique et analogiqce de la langue française, Paris,
Société du Nouveau Littre, 1960-1964, 7 vol. 14. RORBET, P., Petit Robert ou Dictionnaire alphabétique et analogique de la langue
française, Paris, Société du Nouveau Littre, 1978, 2172 p.

15. SECRETARIAT D'ETAT, Bureau des traductions, Termes fiscaux, financiers et administratifs, (Bulletin de terminologie, 154), Ottawa, 1978, 284 p. (s.v. holder).

16. SYLVAIN, F., Dictionnaire de la comptabilité, Canada, Institut canadien des comp-
tables agréses, 1977, 258 p.

17. THOMAS, A.V., Dictionnaire des difficultés de la langue française, Paris, Larousse,
1974, 435 p.

18. VAN HOOF, H., Terminologie économique, Paris, Dunod, 1967, 771 p.

19. Comité consultatif international télégraphique et téléphonique, Exploitation et tarification télégraphiques, Livre orange, t. II.3, Genève, Union internationale des télécom-
munications, 1977, 229 p. (v. p. 114 et 115).

20. Postes et Télécommunications, Service téléphonique, Manuel d'enseignement profes-
sionnel, tome III-B, Paris, Imprimerie sionnel, tome III-B, Paris, Imprimerie Nationale, 1968, p. 190. 


\section{LISTE SELECTIVE DES COOCCURRENTS}

Voici une liste de cooccurrents qui s'accollent à chacun des termes étudiés plus haut.

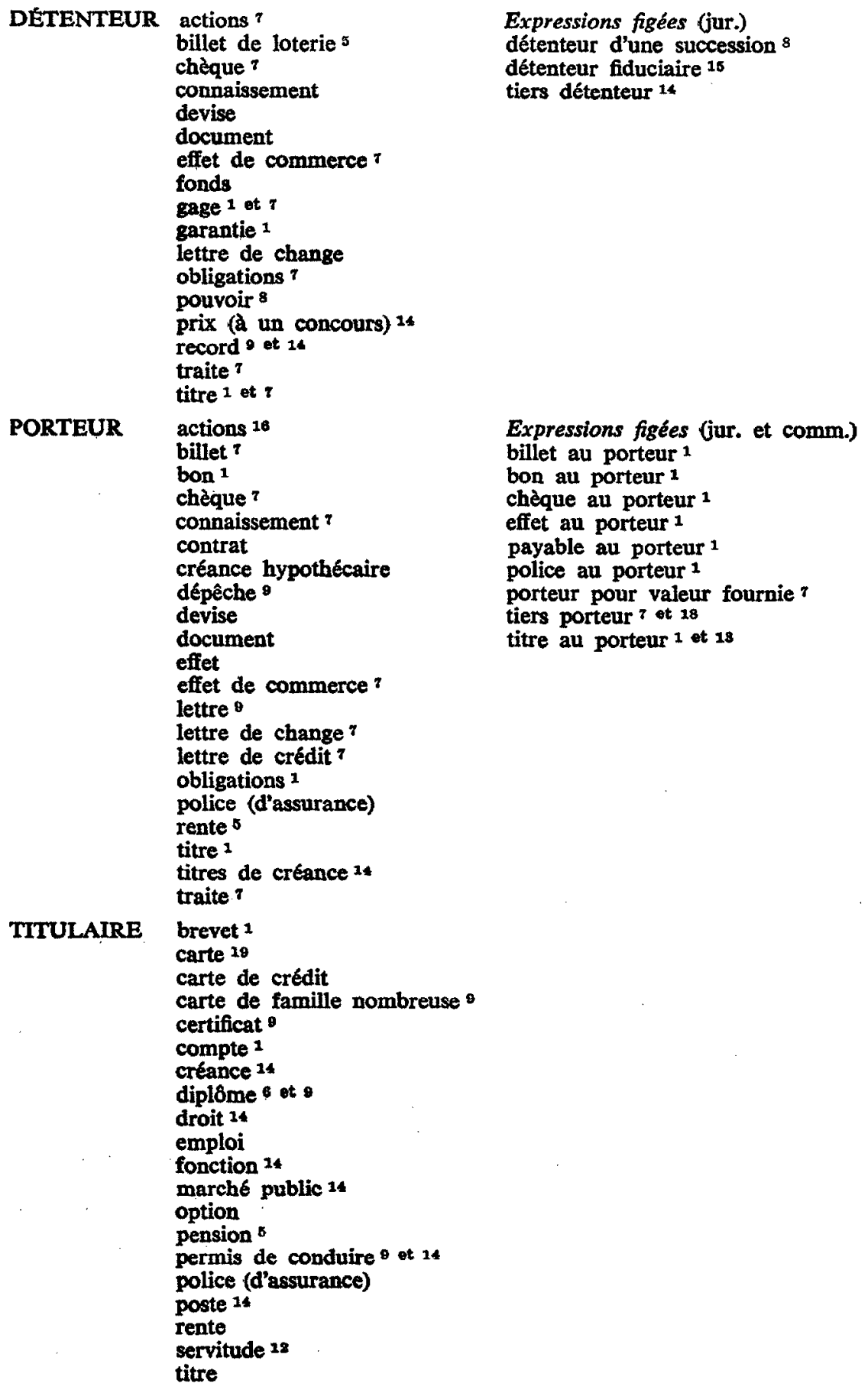

Expressions figées (jur. et comm.)

billet au porteur 1

bon au porteur 1

chèque au porteur 1

effet au porteur 1

payable au porteur ${ }^{1}$

police au porteur ${ }^{1}$

porteur pour valeur fournie?

tiers porteur 7 ot 18

titre au porteur 1 et 18 